\title{
Mathematical Modeling of Data Based on the Demand of Emergency Services of Hospital in order to Maximize the Coverage and Minimize the Operational Costs
}

\begin{abstract}
Introduction: In today's high-traffic world, where humans are constantly confronted with various dangers around the world, a proper response system with the proper functioning of prehospital medical emergencies is of paramount importance. A major challenge for emergency services worldwide is dispatching ambulances to patients appropriately in terms of limited resources and patient safety.

Methods: The mathematical model was first introduced based on the ambulance distribution process in East Tehran Emergency Area and solved using GAMS software based on Epsilon constraint technique and Pareto model analysis. The objective functions are to maximize coverage and minimize costs. To deal with the uncertainties of the parameters, a robust optimization approach was used and the Epsilon method was used for two purposes of modeling. To clarify the model and illustrate the application of the above mathematical model in the real world, a case study was conducted in the eastern region.

Results: Based on the proposed model, the number of stations in East Tehran can be reduced from 39 to 19 , but the number of ambulances in each station is almost twice as high as the average of 4 or 5 ambulances per station.

Conclusion: Reducing the number of bases can have a significant impact on the cost of stationary station equipment, personnel amenities, the cost of time consumed and the property used to build the station, thus saving more stations to reduce time. Responding to demands established increased productivity.
\end{abstract}

Keywords: Linear programming model, Emergency medical system, Constraints, Uncertainty, Reliability, Maximization of cover, Minimization of operational costs.

\footnotetext{
Affiliations

1- PhD student, Department of Industrial Management, Tehran North Branch, Islamic Azad University, Tehran, Iran

2- Associate Professor, School of Industrial Engineering, Iran University of Science and Technology, Tehran, Iran

Corresponding Author: pishvaee@iust.ac.ir

3- Assistant Professor, Department of Anesthesiology, Imam Khomeini Hospital Complex, Tehran University of Medical Sciences, Tehran, Iran 4- Assistant Professor, Department of Industrial Management, Faculty of Management, Tehran North Azad University, Tehran, Iran
}

Article Info

Authors:

Mahmoodi $\mathrm{H}^{1} \oplus$

Pishvaee $\mathrm{MS}^{2^{*} \text { ๑ }}$

Saberian $\mathrm{P}^{3 \odot}$

Shoar $\mathrm{M}^{4} \bullet$

Received: 2020/7/3

Accepted: 2020/8/18

E-Published: 2020/9/5

\section{Citation Subjects:}

EMS, Critical Care, First Aid, Hot-lines, Programming, Mathematics, Coverage, Affordability, Cost-Minimization Analysis, Pricing, Cost Comparison 
فصلنامه يرستار و يزشك در رزم / تابستان 99 / سال هشتم

مدلسازى رياضى دادهها بر اساس تقاضاى خدمات اورزانس ييشبيمارستانى به منظور بيشينهسازى ميزان يوششدهى و كمينهسازى هزينهاى عملياتى

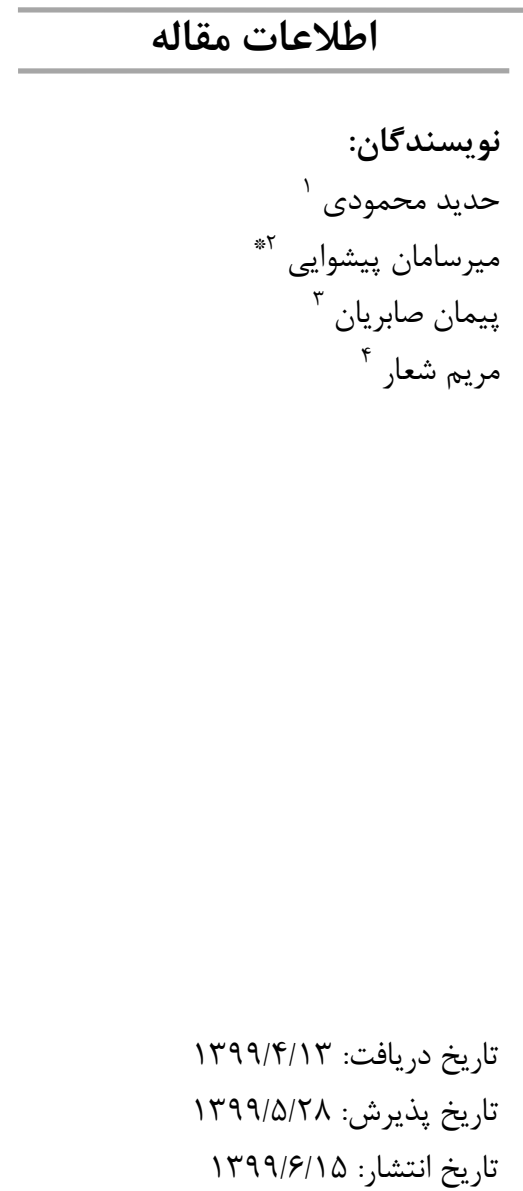

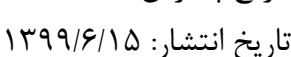

موازگًان كليدى: مدل برنامهريزى خطى، عدم قطعيت، قابليت اطمينان، بيشينهسازى كمينه

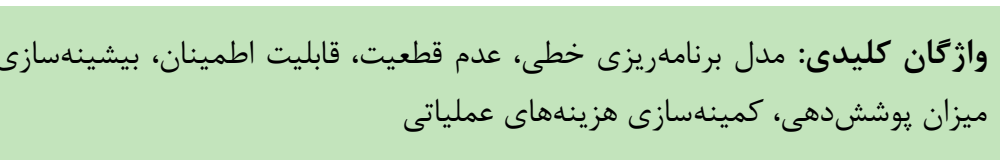

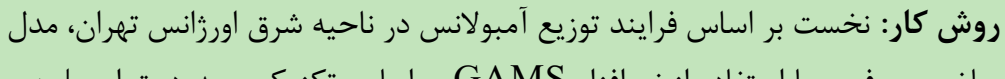

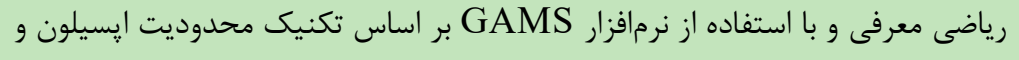

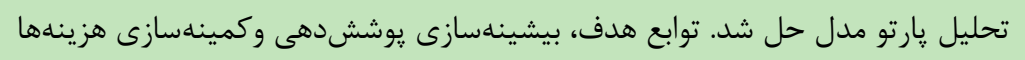

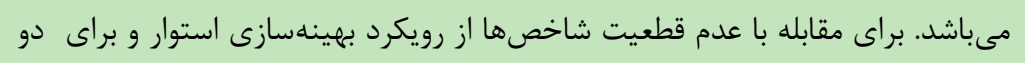

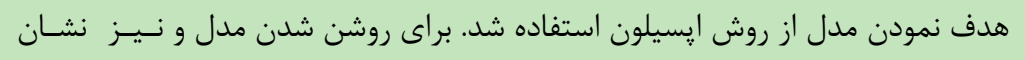

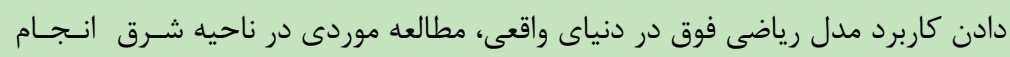
يافت.

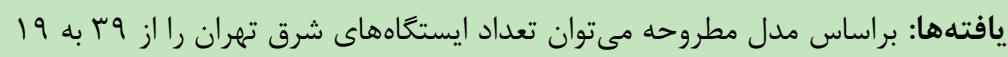

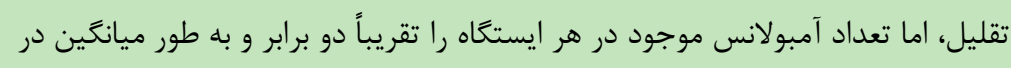

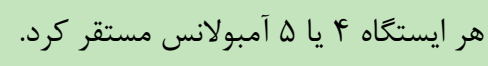

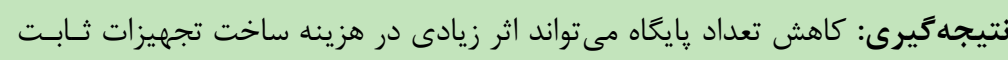

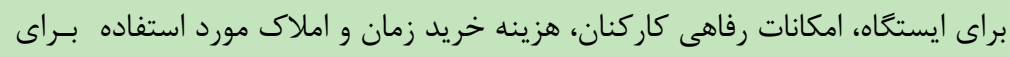

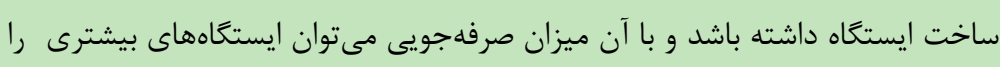

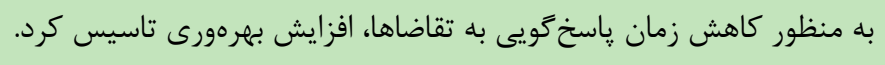

$$
\text { وابستكى سازمانى نويسندكان }
$$

ا - دانشجوى دكترى تخصصى، تروه مديريت صنعتى، دانشعاه آزاد اسلامى واحد تهــران شــــال،

عناوين ارجاعى: يوشش، حداكثر سازى، بزرى كردن، ارن، مخارج، تقليل
Pishvaee@iust.ac.ir (نويسنده مسئول)

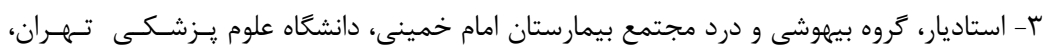
تهران، ايران F - استاديار، كروه مديريت صنعتى، دانشعاه آزاد اسلامى واحد تهران شمال، تهران، ايران 


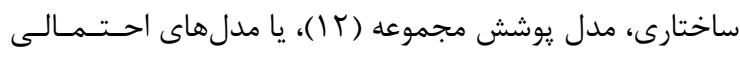

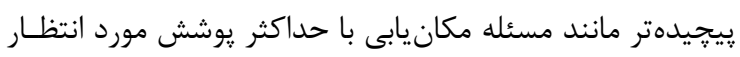

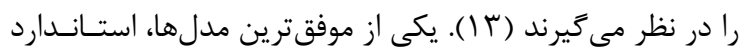

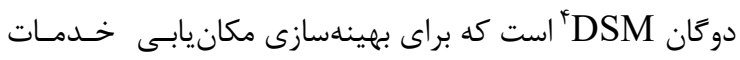

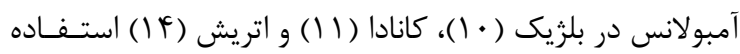

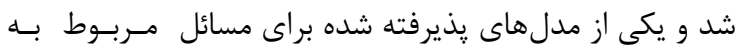

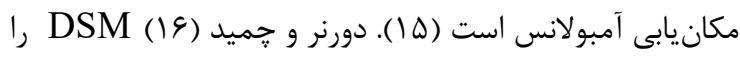

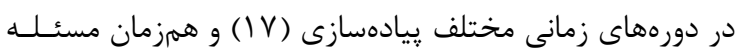

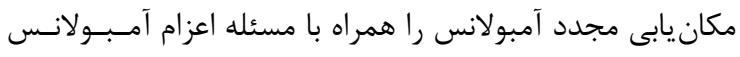

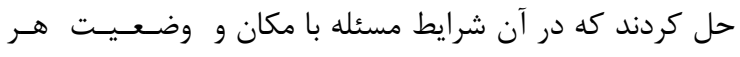

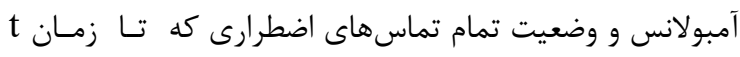

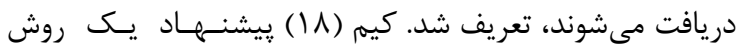

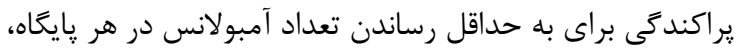

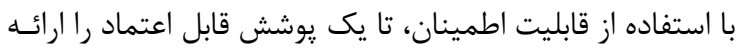

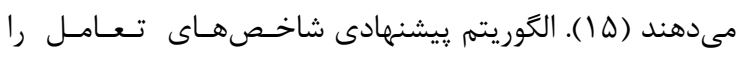

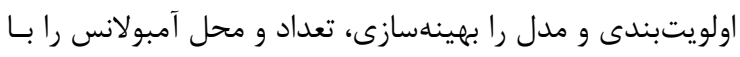

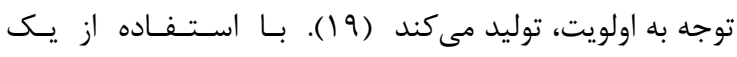

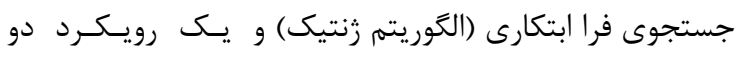
مرحلهاى با در نظر داشتن تقسيمبندى منطقه سرويس و تعـداد واحدها در هر يارتيشن همزمان، بر اساس مدل صف، اين مسئله را حل مى كند.

روش كار

تحقيق حاضر، يك مطالعه كاربردى - توسعهاى بود و به صورت

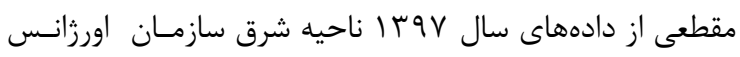

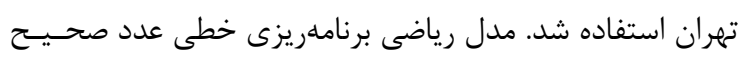
مختلط جهت انتخاب روش حل مسئله بهينهسازى ايستخاههاى

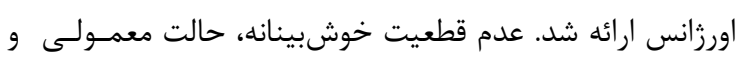

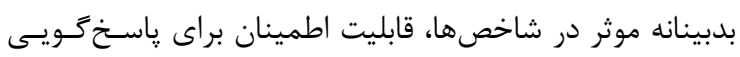

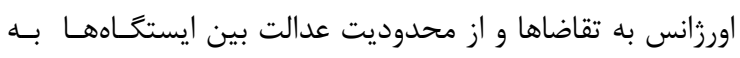

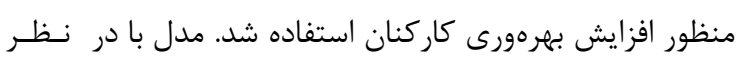

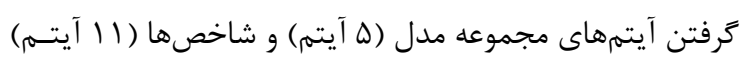

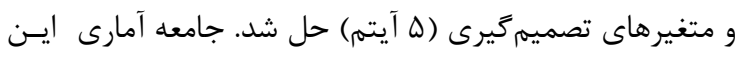
يروهش، كليه دادهاى بانك ديتاى ناحيه شرق (9 ماه دوم سال

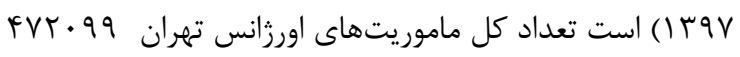

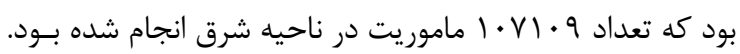

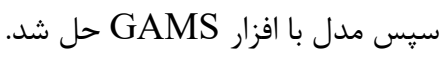

يافتهها

در هر مرحله يكى از تابع هدفها حل و ساير توابع بـهـ صـورت محدوديت در مدل اعمال شد.

4- Double Standard Model
مقدمه

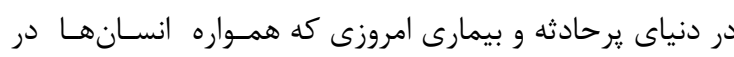

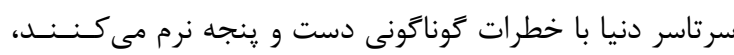

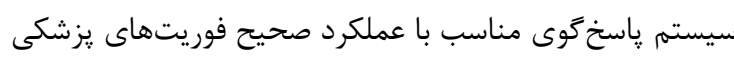

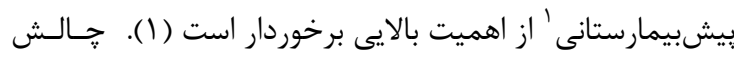

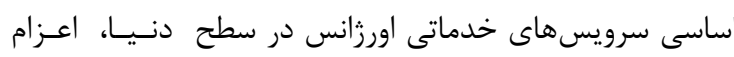

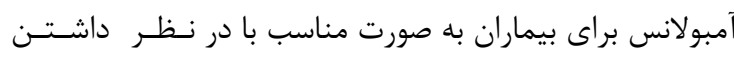

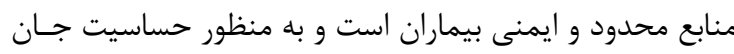

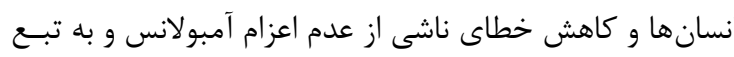

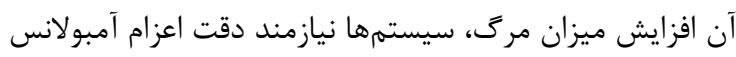

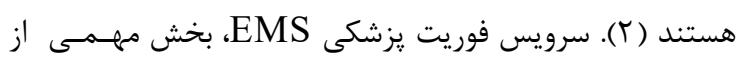

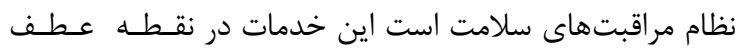
مراقبتهاى سلامت، سلامت عمومى و ايمنى فعاليت مى نمايسـد.

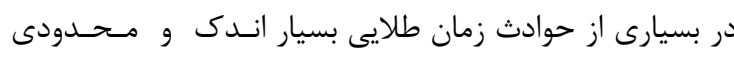

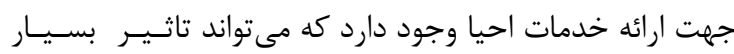

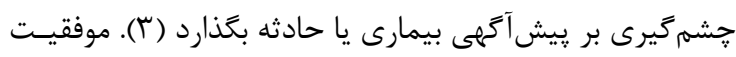

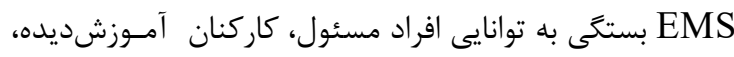

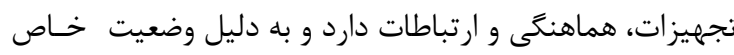

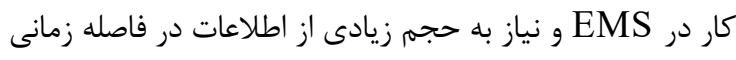

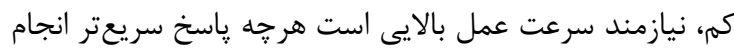
شود، نتيجه بهترى براى بيمار به بار مى آيد (1).

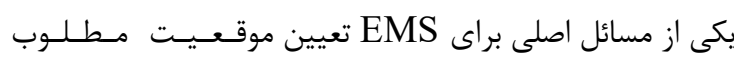

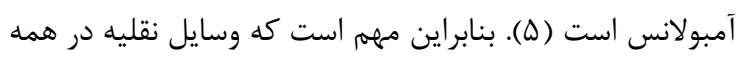

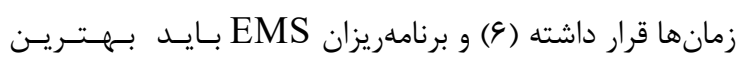
مكان ها را براى آمبولانسها دما تعيين كنند (V) (V). محققين دريافتند

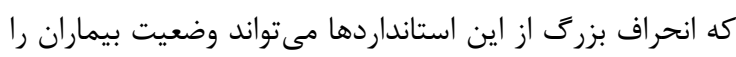

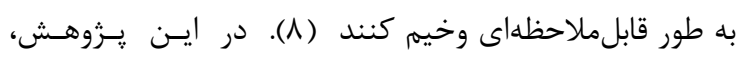
مسئلهى مكانيابى - تخصيص استوار سايتهاى اورزانس ناحيه

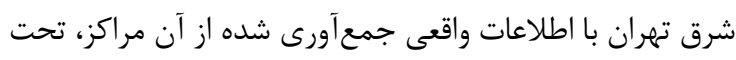

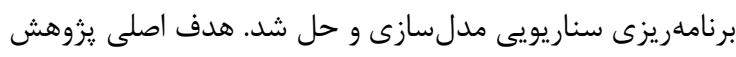

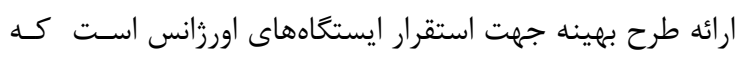

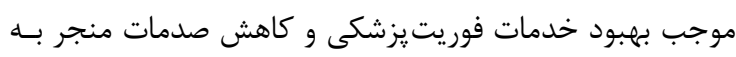

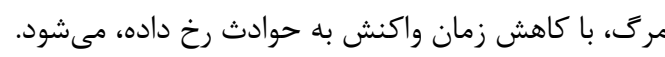

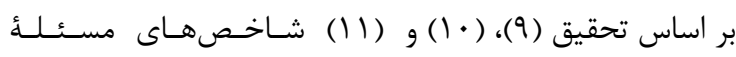

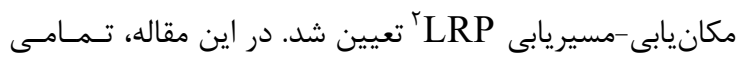

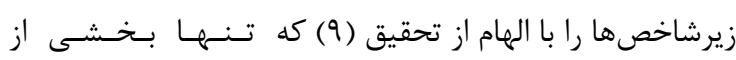

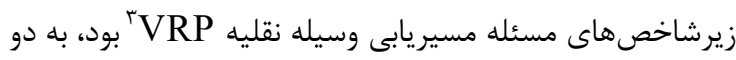

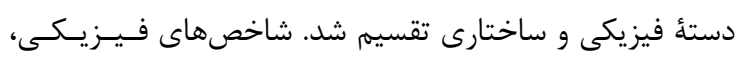

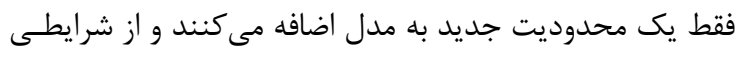

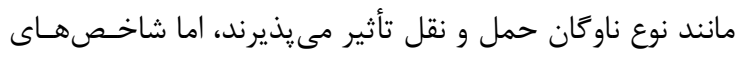

1- Emergency Medical System - EMS

2- Location-routing problem - LRP

3- Vehicle routing problem - VRP 
جدول ا: مجموعههاى استفاده شده در مدل رياضى

\begin{tabular}{|c|c|}
\hline تعريف & مجموعه \\
\hline 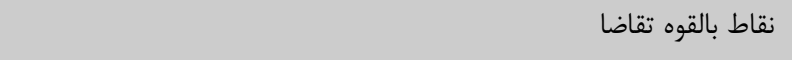 & $i$ \\
\hline نقاط بالقوه براى تأسيس ايستخاه & $j, j^{\prime}$ \\
\hline سناريوهاى زمانى & S \\
\hline 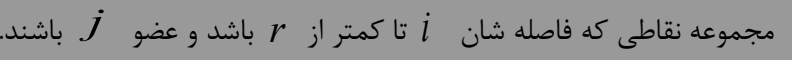 & $w_{i}^{r}$ \\
\hline مجموعه سناريوهاى عدم قطعيت & $K$ \\
\hline
\end{tabular}

جدول r: يار امترها و متغيرهاى مدل رياضى

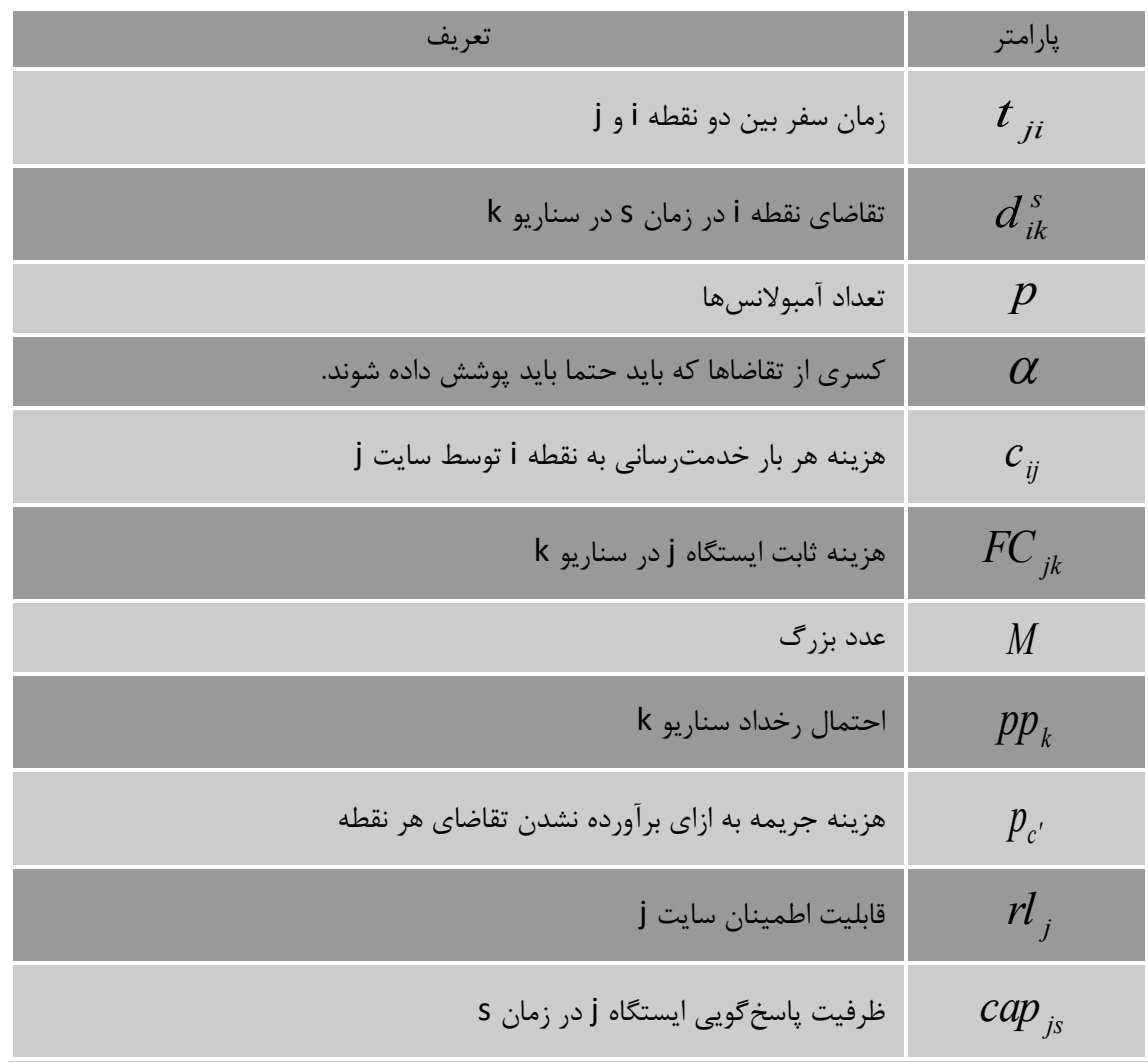

\begin{tabular}{|c|c|}
\hline تعريف & متغير تصميمثيرى \\
\hline تعداد آمبولانسهاى سايت ز در سناريو k & $x_{j k}$ \\
\hline 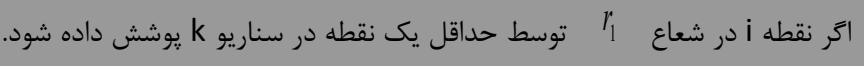 & $y_{i k}$ \\
\hline احر نقطه أ در شعاع $1 r_{1}$ توسط حداقل دو نقطه در سناريو k يوشش داده شود. & $z_{i k}$ \\
\hline اكر سايت ز در سناريو k فعال باشد. (تأسيس شود) & $R_{j k}$ \\
\hline احر تقاضاى i در زمان S در سناريوى k توسط سايت j ياسخ داده شود. & $Q_{i j k}^{s}$ \\
\hline
\end{tabular}




\begin{tabular}{|c|c|c|}
\hline & \multicolumn{2}{|c|}{ مدل رياضى يزوهش } \\
\hline $\max \mathrm{z}_{1}=\sum_{i} \sum_{s} \sum_{k} d_{i k}^{s} \times \mathrm{Q}_{i j k}^{s} \times p p_{k}$ & & 1 \\
\hline $\begin{array}{l}\min \mathrm{z}_{2}=\sum_{j} \sum_{k} F C_{j k} \times R_{j k} \times p p_{k}+\sum_{i} \sum_{j} \sum_{s} \sum_{k} c_{i j} \times d_{i k}^{s} \times Q_{i j k}^{s} \times p p_{k} \times r l_{j} \\
+\sum_{i} \sum_{j} \sum_{k} \sum_{s} Q_{i j k}^{s}\left(1-r l_{j}\right) \times p_{c^{\prime}}\end{array}$ & & $r$ \\
\hline$\sum_{j \in w_{i}^{r 1}} x_{j k} \geq 1$ & $\forall \mathrm{i}, \mathrm{k}$ & r \\
\hline$\sum_{i} d_{i s}^{k} y_{i k} \geq \alpha \sum_{i} d_{i k}^{s}$ & $\forall \mathrm{s}, \mathrm{k}$ & r \\
\hline$\sum_{j \in w_{i}^{r_{2}}} x_{j k} \geq \mathrm{y}_{i k}+\mathrm{z}_{i k}$ & $\forall \mathrm{i}, \mathrm{k}$ & $\Delta$ \\
\hline$z_{i k} \geq y_{i k}$ & $\forall \mathrm{i}, \mathrm{k}$ & 4 \\
\hline$\sum_{j \in w} x_{j k}=p$ & $\forall \mathrm{k}$ & v \\
\hline$x_{j k} \leq R_{j k} \times \mathrm{M}$ & $\forall \mathrm{j}, \mathrm{k}$ & $\wedge$ \\
\hline$Q_{i j k}^{s} \leq \mathrm{R}_{j k}$ & $\forall \mathrm{i}, \mathrm{j}, \mathrm{k}, \mathrm{s}$ & 9 \\
\hline$\sum_{j} \sum_{s} Q_{i j k}^{s} d_{i k}^{s} \geq 1$ & $\forall \mathrm{i}, \mathrm{k}$ & 1. \\
\hline$\sum_{i} Q_{i j k}^{s} d_{i s}^{k} \leq x_{j k} \operatorname{cap}_{j s}$ & $\forall \mathrm{j}, \mathrm{k}, \mathrm{s}$ & 11 \\
\hline$x_{j k} \leq c a p_{j s}$ & $\forall \mathrm{j}, \mathrm{k}, \mathrm{s}$ & ir \\
\hline
\end{tabular}

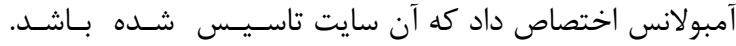

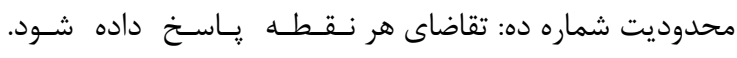

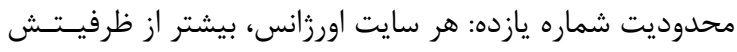

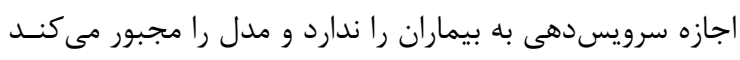

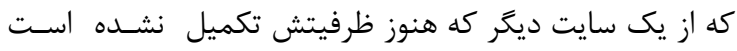

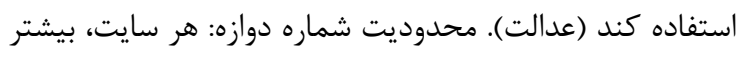

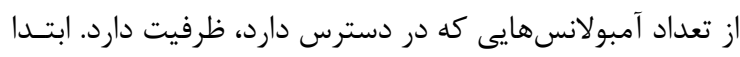

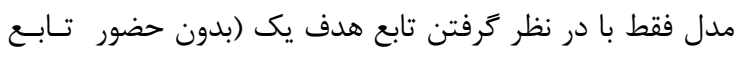

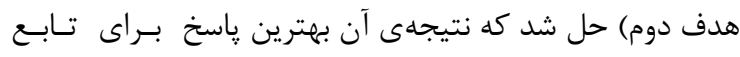

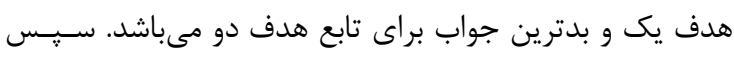

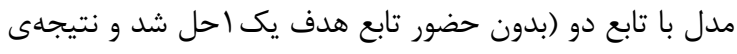

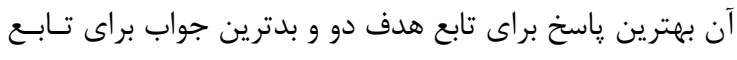

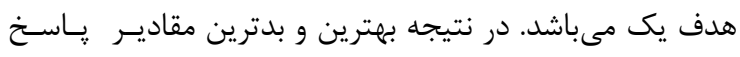
براى تابع هدفهاى يك و دو به دست آمد.
تابع هدف شماره يك: سعى بر حداكثر كردن ميزان تـقــاضـاى

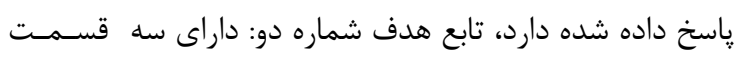

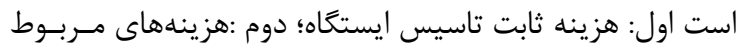

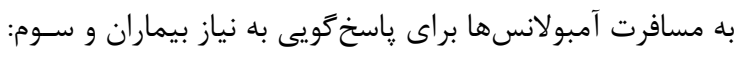

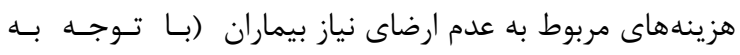

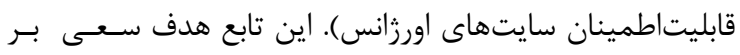

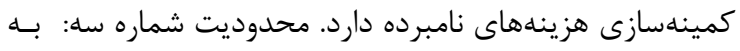

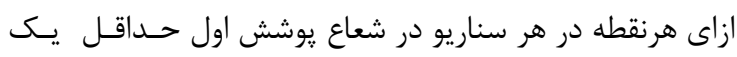

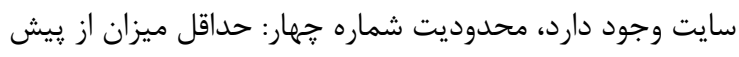

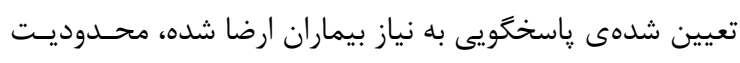

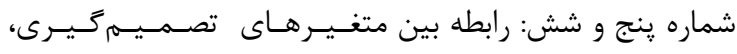

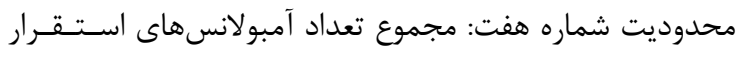

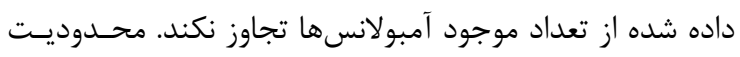

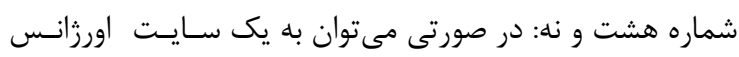




\section{جدول سّ: ياسخهاى هر دو تابع هدف}

\begin{tabular}{|c|c|c|}
\hline مقدار & تابع هدف | (نقطه) & تابع هدف ץ (هزار تومان) \\
\hline بهترين جواب & rolf & $|r r \Delta q|$. \\
\hline بدترين جواب & NT & $r q Y \Delta Q \Delta \Lambda$ \\
\hline
\end{tabular}

شكست مى دهيم (مثلاً ه نقطه). طول بازهى بهترين تا بدتريسن

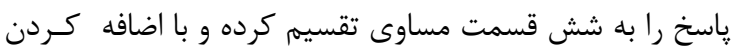
عدد به دست آمده به بهترين ياسخ، مقدار تابع هدف دو دو در اين رين نقاط به دست مى آيد.
جدول فوق ياسخ بهينه كل مسئله مربوط به تابع هدف يك در

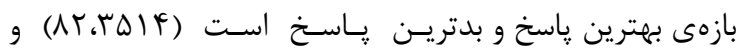

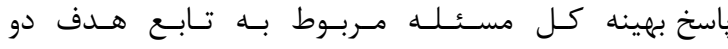

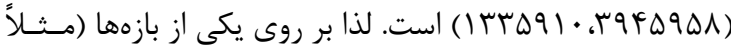

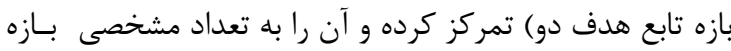

جدول Fٔ: مقدار تابع هدف † در نقاط شكست

\begin{tabular}{|c|c|}
\hline نقطه & نابع هدف r \\
\hline 1 & $18 V \cdot 911$ \\
\hline$r$ & $r r \cdot \Delta q r \Delta$ \\
\hline r & TGF. QTr \\
\hline c & $r \cdot v \Delta q r q$ \\
\hline$\Delta$ & $r \Delta 1.949$ \\
\hline
\end{tabular}

تابع هدف يك به دست آورده شود.

تابع هدف يك در مدل رياضى را به محدوديت منتقل كـرده و

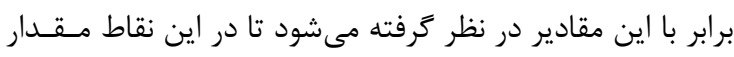

\section{جدول ه: مقدار تابع هدف ا در نقاط شكست}

\begin{tabular}{|c|c|c|}
\hline نقطه & مقدار تابع هدف دو در اين نقطه (هزار تومان) & مقدار تابع هدف يك در اين نقطه (نقطه) \\
\hline بهترين پاسخ تابع هدف دوم & $\mid r r \Delta q 1$. & Ar \\
\hline 1 & $1 V V \cdot 911$ & $1 \cdot r$ \\
\hline r & $r T \cdot \Delta q T \Delta$ & rA. \\
\hline r & rgF. qur & $\wedge \cdots$ \\
\hline f & $r \cdot \vee \Delta q r q$ & IQHA \\
\hline$\Delta$ & rol. qfe & relf \\
\hline بدترين پاسخ تابع هدف دوم & $r q 4 \Delta 9 \Delta \Lambda$ & rolf \\
\hline
\end{tabular}

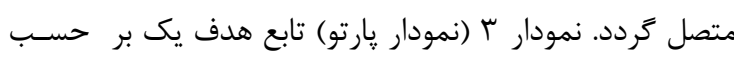

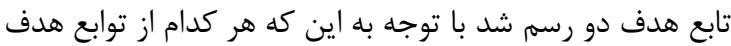

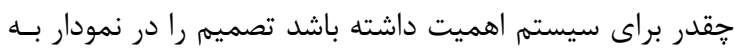

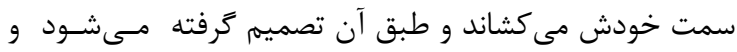

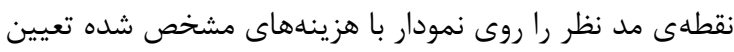

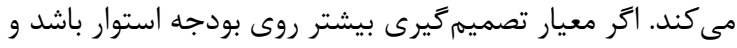

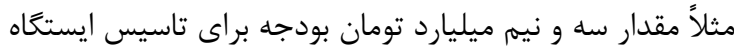

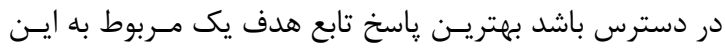

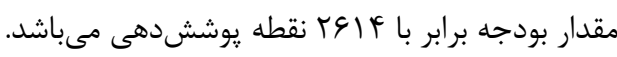

در نمودار شماره يك و دو ميزان تاثير تغييرات قابليت اطمينان

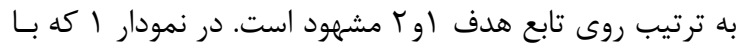

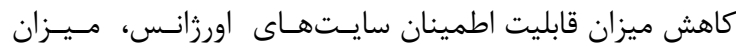
يوشش نقاط (تابع هدف يك) توسط اين سايت با شيب كمترى

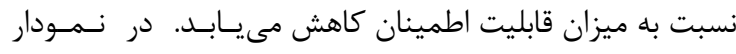

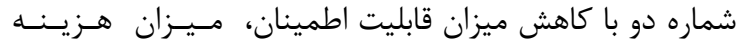

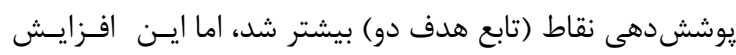

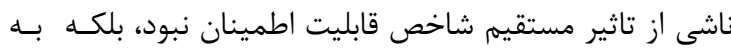

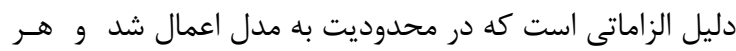

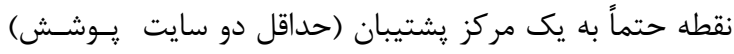




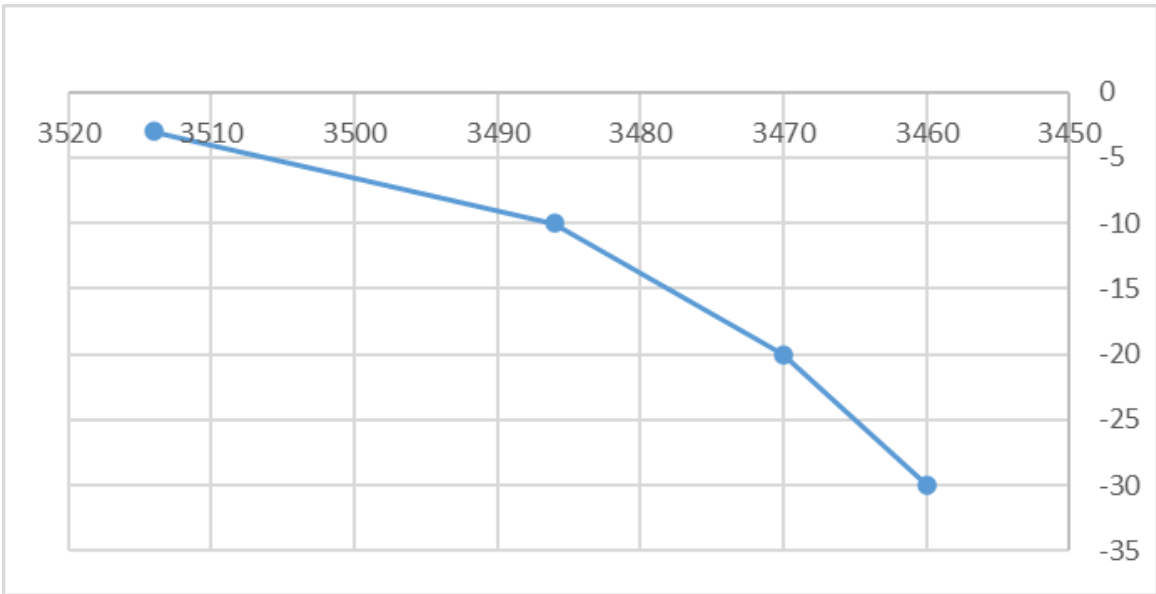

نمودار ا: ميزان حساسيت تابع هدف يك به قابليت اطمينان

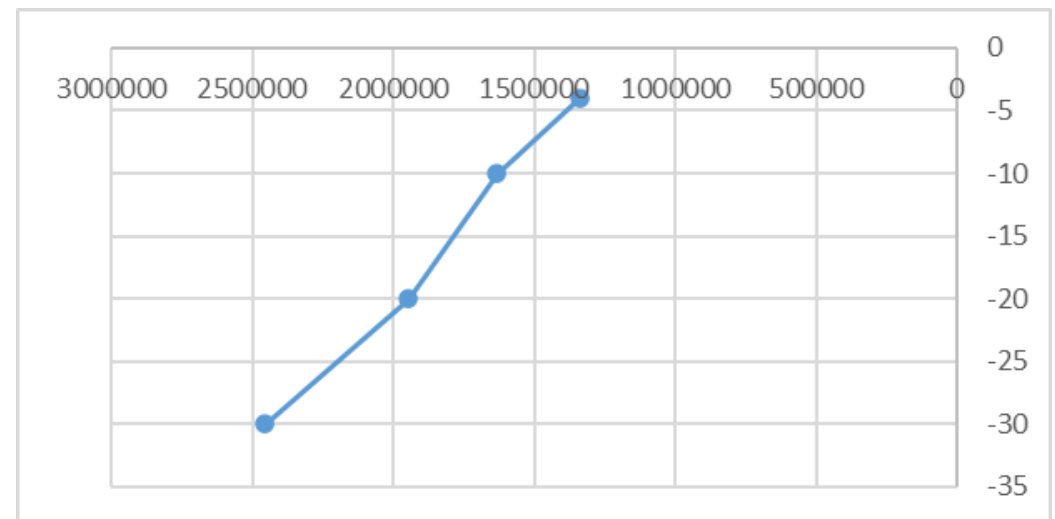

نمودار r: ميزان حساسيت تابع هدف دو به قابليت اطمينان

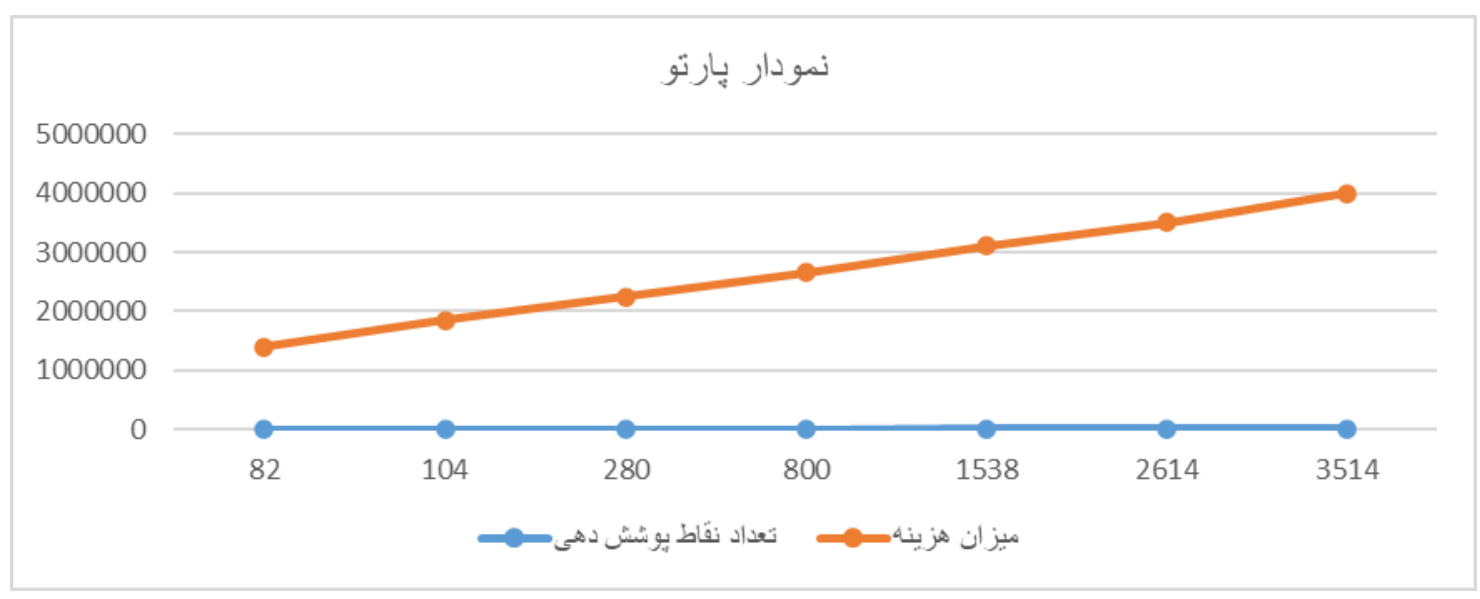

نمودار بّ: پِارتو بر آورد ميزان هزينه و تعداد نقاط يوششدهى 


\section{References}

1- Dami FGPFCH. Prehospital triage accuracy in a criteria based dispatch centre. BMC Emergency Medicine. 2019;15(32).

2- Bohm K, Kurland L. The accuracy of medical dispatch - a systematic review. Scandinavian Journal of Trauma Resuscitation and Emergency Medicine December 2018;26(1).DOI: 10.1186/s13049-018-0528-8.

3- Vafaee nasab M, Fateh panah A, Jahangiri K, Namdari M. Evaluation of Yazd Prehospital Emergency Pegasys Performance Based on Duration of Patient Presentation. Toloe behdasht. 2017;15(5):122-31.

4- Zarratchi A, Rostami B, Rostami A. Temporal Characteristics of Pre-hospital Emergency Services; A Cross-sectional Study. Iranian Journal of Emergency Medicine. 2016;5(8).

5- Zhen L, Wang K, Hu H, Chang D. A simulation optimization framework for ambulance deployment and relocation problems. Computers \& Industrial Engineering. 2014(72):12-23.

6- Su Q, Luo Q, Huang SH. Cost-effective analyses for emergency medical services deployment: A case study in Shanghai. International Journal of Production Economics. 2015 (163):112-23.

7- Van den Berg PL, Kommer GJ, Zuzáková B. Linear formulation for the maximum expected coverage location model with fractional coverage. Operations Research for Health Care. 2016;8:33-41.

8- Shariff SR, Moin NH, Omar M. Location allocation modeling for healthcare facility planning in Malaysia. Computers \& Industrial Engineering. 2012;62(4):1000-10.

9- Laporte G, Nickel S, Saldanha da Gama F. Location science: Springer; 2016.

10- Simon D. Biogeography-based optimization. IEEE transactions on evolutionary computation. 2008;12(6):702-13.

11- Mansouri N, Mohammad Hasani Zade B, Javidi MM. Hybrid Task Scheduling Strategy for Cloud Computing by Modified Particle Swarm Optimization and Fuzzy Theory. Computers \& Industrial Engineering. 2019;130:597 $-633$.

12- Atta S, Mahapatra PRS, Mukhopadhyay A. Solving maximal covering location problem using genetic algorithm with local refinement. Soft Computing. 2018;22(12):3891-906.

13- Cordeau JF, Furini F, Ljubić I. Benders decomposition for very large scale partial set covering and maximal covering location problems. European Journal of Operational Research. 2019;275(3):882-96.

14- Derrac J, García S, Molina D, Herrera F. A practical tutorial on the use of nonparametric statistical tests as a methodology for compar-

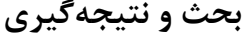

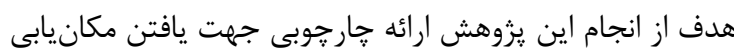

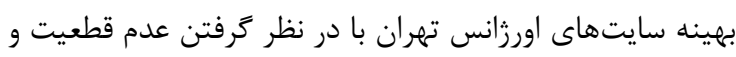

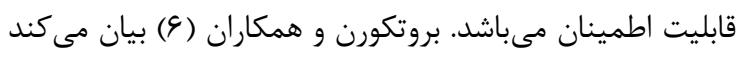

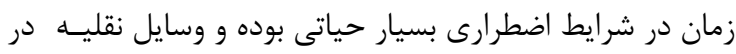

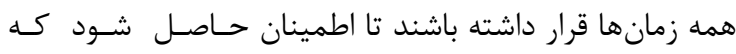

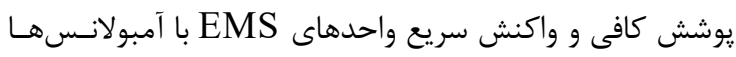

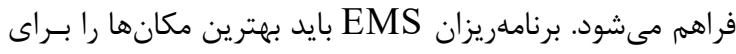

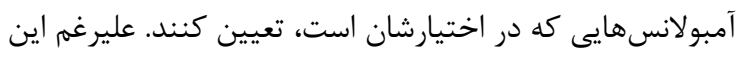

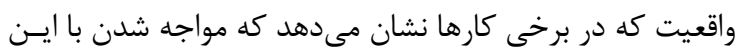

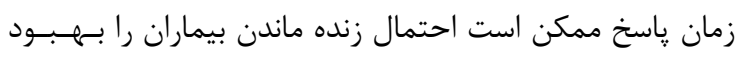

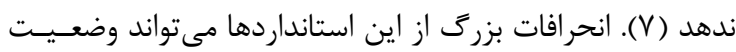

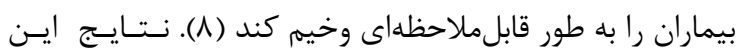

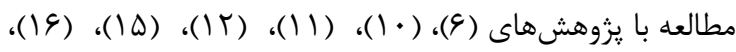

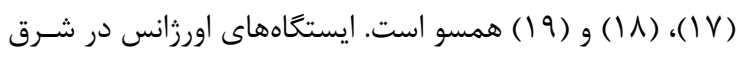

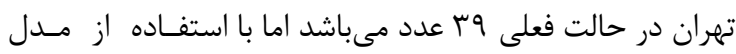

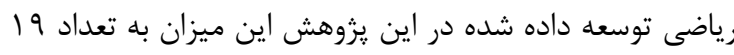

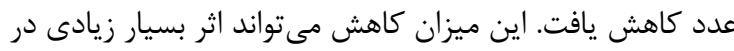

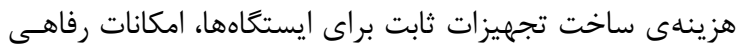

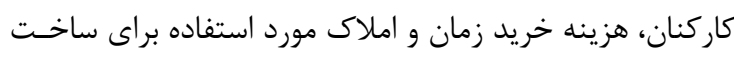

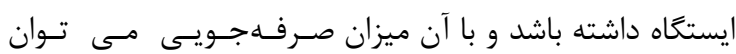

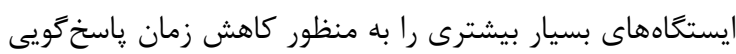

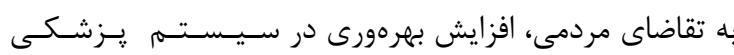

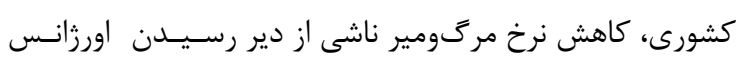

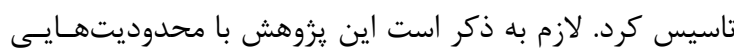

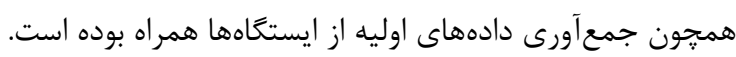

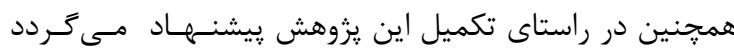

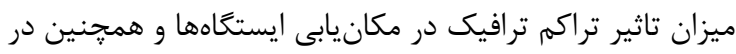

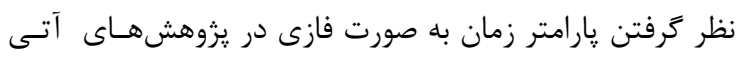

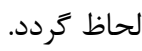
تشكر و قدردانى لحزي نويسندكان مقاله بر خود لازم مي دانند از همكارى و مســاعـدت

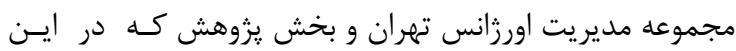
يزولي ما را يارى نمودند سياسكزارى نمايند. 
ing evolutionary and swarm intelligence algorithms. Swarm and Evolutionary Computation. 2011;1(1):3-18.

15- Rodriguez FJ, Blum C, Lozano M, GarcíaMartínez C, editors. Iterated greedy algorithms for the maximal covering location problem. In European Conference on Evolutionary Computation in Combinatorial Optimization; 2012; Berlin: Springer.

16- Senne ELF, Pereira MA, Lorena LAN. A decomposition heuristic for the maximal covering location problem. Advances in Operations Research. 2010.

17- Zarandi MF, Davari S, Sisakht SH. The large scale maximal covering location problem. Scientia Iranica. 2011;18(6):1564-70.

18- Davari S, Zarandi MHF, Hemmati A. Maximal covering location problem (MCLP) with fuzzy travel times. Expert Systems with Applications. 2011;38(12):14535-41.

19- Helle KB, Pebesma E. Optimising sampling designs for the maximum coverage problem of plume detection. Spatial Statistics. 2015 (13):21-44. 